Recent Insights into the Physics of the Sun and Heliosphere:

Highlights from $\mathrm{SOHO}$ and Other Space Missions

IAU Symposium, Vol. 203, 2001

P. Brekke, B. Fleck, and J. B. Gurman eds.

\title{
Looking for the FIP Effect in EUV Spectra
}

\author{
B. N. Dwivedi
}

Dept. of Appl. Phys., Banaras Hindu Univ., Varanasi-221005, India

E. Landi

Max-Planck-Institut für Aeronomie, 37191 Katlenburg-Lindau, Germany

A. Mohan

Dept. of Appl. Phys., Banaras Hindu Univ., Varanasi-221005, India

\begin{abstract}
We present results from a study of extreme-ultraviolet (EUV) off-limb spectra obtained with the SUMER instrument on the spacecraft SOHO. Using EUV line intensities, we deduce plasma temperatures and densities in the off-limb solar plasma. We make use of this information to study the FIP effect in the solar corona. We have looked for FIP effect in EUV spectra obtained by SUMER in a considerable detail. In particular, we report $\mathrm{K} / \mathrm{Ar}, \mathrm{Si} / \mathrm{Ar}$ and $\mathrm{S} / \mathrm{Ar}$ relative element abundances and investigate the height dependence of the FIP bias in the solar corona. Also, we study the relative $\mathrm{Mg} / \mathrm{Ne}$ abundance in an active region at the solar limb to investigate the correlation of the FIP bias with magnetic loop structures in the field of view.
\end{abstract}

\section{Introduction}

Elemental abundances are among the most fundamental sets of parameters in the solar and stellar atmospheres. There has lately been vast literature on the topic, including the most recent review by Feldman and Laming (2000). The status of coronal abundances relative to hydrogen is not entirely settled: there is a significant body of evidence that abundances are correlated with the first ionization potential, giving rise to the so-called FIP effect. This effect consists of systematic differences between photospheric and coronal element abundances. The latter show enhancements of a factor around 4 in the low-FIP elements (FIP $<10 \mathrm{eV}$ ), while abundances for the high-FIP elements (FIP $>10 \mathrm{eV}$ ) remain constant between the photosphere and the corona. There is no theoretical model at present able to explain this effect satisfactorily.

Dwivedi et al. (1999) carried out an observing sequence based on a theoretical study by Dwivedi and Mohan (1995), with intercombination/ forbidden $\mathrm{Ne}$ VI and $\mathrm{Mg}$ VI lines, formed at essentially the same temperature $\left(4 \times 10^{5}\right.$ $\mathrm{K})$. The FIPs of $\mathrm{Ne}$ and $\mathrm{Mg}$ are 21.6 and $7.6 \mathrm{eV}$, respectively: they form a high-FIP/low-FIP pair. This study provided new observational facts in transition region emission lines (Dwivedi et al. 1999). In the present paper, we extend 


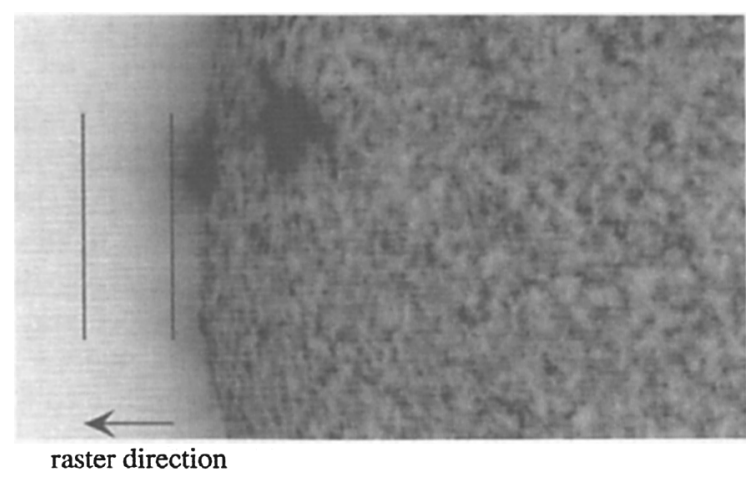

Figure 1. He II map of the region with the limits of the SUMER raster superimposed.

tion region emission lines (Dwivedi et al. 1999). In the present paper, we extend this investigation taking account of other low-FIP/high-FIP pairs such as $\mathrm{K} / \mathrm{Ar}$, $\mathrm{Si} / \mathrm{Ar}$ and S/Ar present in the spectra. The use of the recently identified K XIII line at $994.58 \AA$ (Mohan et al. 2000a) allows us to investigate the amount of FIP bias also for the very low-FIP element $\mathrm{K}(\mathrm{FIP}=4.3 \mathrm{eV})$, so that it is possible to investigate whether the FIP bias of the low-FIP elements is constant or depends on the FIP value of each element, as suggested by Feldman and Laming (2000). Also, the $\mathrm{Mg} / \mathrm{Ne}$ ratio is re-examined and correlated with magnetic structures in the field of view. More details on this investigation can be found in Mohan et al. (2000b).

\section{Observations and Data Analysis}

The observations were made with the SUMER spectrograph on 1996 June 20 above the active region NOAA 7974 at the solar east limb. Fig. 1 shows the position and extension of the SUMER raster superimposed on the He II $304 \mathrm{~A}$ image taken with the EIT telescope. The observations analyzed in the present work include both plasma from an active region at the limb and plasma outside the structures of the active region.

The Mg VI intensity map shows a highly structured behaviour, indicating that this ion is present only inside relatively cool magnetic structures in the active region. Outside, the residual $\mathrm{Mg}$ VI emission is due to scattered light. S X, on the contrary, shows no signature of the active region, and presents a fairly uniform map with intensity decreasing with distance from the limb. It is, therefore, reasonable to assume that the plasma generating the coronal ions is completely unrelated to the Ne VI and Mg VI cool structures. For this reason, in the present work these two plasmas have been analyzed separately.

Making use of density-sensitive $1190 / 1191 \mathrm{Mg}$ VI line ratio, we find the

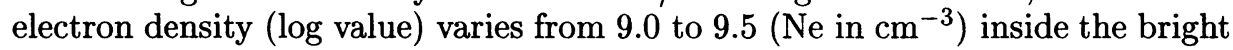
structures. The temperature, determined via the $\mathrm{Mg}$ VII/Mg VI line ratio, is almost constant, with a value of about $5.6 \times 10^{5} \mathrm{~K}$. Density and temperature for the off-limb plasma have been determined via 1196/1213 S X and Ar XII 1018/S 
$\mathrm{X}(1196+1213)$ ratios respectively. While $\log \mathrm{N}_{e}$ is fairly low (7.0 to 8.2$)$, the temperature remains almost constant with height with $\log \mathrm{T} \simeq 6.25(\mathrm{~T}$ in $\mathrm{K})$.

Making use of the intensity maps from Ne VI $1005.70 \AA$ and Mg VI 1191.64 $\AA$, we determined the $\mathrm{Ne} / \mathrm{Mg}$ ratio across the whole field of view and compared it with the Ne VI $1005.70 \AA$ intensity map. The variation of the $\mathrm{Mg} / \mathrm{Ne}$ relative abundances is strongly correlated with plasma structures in the emitting source. Although the instrument resolution is not enough to resolve individual structures precisely, we find that some structures with strong Ne VI and Mg VI emission indicate a normal $\mathrm{Mg} / \mathrm{Ne}$ FIP-bias (1.6 to 3.2). In regions with strong $\mathrm{Mg}$ VI and weak Ne VI emission the FIP-bias ranges from 3.1 to 8.8. These values show that the plasma structuring in active regions has a strong effect on element abundances.

In the unstructured, hot coronal plasma we studied the low-FIP/high-FIP pairs $\mathrm{S} / \mathrm{Ar}, \mathrm{Si} / \mathrm{Ar}$ and $\mathrm{K} / \mathrm{Ar}$ and the height dependence of their intensity ratios. The FIPs for $\mathrm{K}, \mathrm{Si}, \mathrm{S}$ and $\mathrm{Ar}$ are 4.3, 8.2, 10.4 and $15.8 \mathrm{eV}$ respectively. The coronal relative abundance of $\mathrm{S} / \mathrm{Ar}$ stays constant at its photospheric value. $\mathrm{Si} / \mathrm{Ar}$ varies between 18 and 26 compared to its photospheric value of 9 . Thus we find a FIP factor 2 to 3 for $\mathrm{Si} / \mathrm{Ar}$ in the corona. The case of $\mathrm{K} / \mathrm{Ar}$ is a new result. $\mathrm{K}$ has a very low abundance, apart from having the lowest FIP among the considered elements. We find that the $\mathrm{K} / \mathrm{Ar}$ relative abundance varies from 0.7 to 1.6 in the off-limb plasma, showing a clear, strong FIP bias that changes from 16 to 36 , stronger than any FIP bias result quoted in the literature. Kink et al. (1999) have identified a Si VIII line, coming from highly excited levels, blending K XIII in quiet Sun spectra. In active region plasma such as the present one, K XIII should be dominant, but the Si VIII blend must be considered. This would reduce the FIP bias. Unfortunately, no accurate estimates of the Si VIII intensity are available. It is also interesting to note that the K FIP bias is dependent on the distance from the limb, unlike the other elements.

In conclusion, we find (1) $\mathrm{Mg} / \mathrm{Ne}$ relative abundance to be strongly correlated with magnetic structures, with relative $\mathrm{Mg} / \mathrm{Ne}$ abundance enhancements reaching up to a factor of 8.8 ; (2) suggestions for the FIP bias inside the low-FIP elements' class might be dependent on the FIP value, being higher for the very low FIP element K, in off-limb active region plasma; (3) some evidence of height dependence of the FIP bias for $\mathrm{K}$, while Si and S results seem almost constant with height within uncertainties.

Acknowledgments. The IAU/ESA Travel Award enabled B.N. Dwivedi to present this work at the IAU Symposium 203.

\section{References}

Dwivedi, B.N. Mohan, A., 1995, Solar phys., 157, 135.

Dwivedi, B.N., Curdt, W., Wilhelm, K., 1999, ApJ, 517, 516.

Feldman, U., Laming, J.M., 2000, Phys. Scripta, 61, 222.

Kink, et al., 1999, ApJ, 512, 496.

Mohan, A., Dwivedi, B.N., Landi, E., 2000a, J. Astr. Astroph., in press.

Mohan, A., Landi, E., Dwivedi, B.N., 2000b, A\&A, submitted. 